\title{
Temporary fragmentation of a marginal lake and its effects on zooplankton community structure and organization
}

\author{
Nadai, R. and Henry, $R$. \\ Department of Zoology, Institute of Biosciences, UNESP, \\ CP. 510, CEP 18618-000, Botucatu, SP, Brazil \\ *email: rhenry@ibb.unesp.br \\ Received February 6, 2008 - Accepted March 24, 2008 - Distributed August 31, 2009
}

(With 5 figures)

\begin{abstract}
A river lateral lake (Coqueiral Lake marginal to Paranapanema River in its mouth zone into Jurumirim Reservoir, São Paulo, Brazil) presented fragmentation into four small isolated bodies of water during a prolonged drought period, disrupting the link with the river. The aim of this work was to compare the temporal modifications on zooplankton community structure (total abundance, species richness, and diversity) in the four water bodies. Zooplankton samplings and abiotic factor measurements were made in two periods - during isolation phase of the lake in relation to river and after re-establishment of hydrologic connectivity. A concentration effect on zooplankton abundance was recorded with drought progression, but without significant modifications in species richness and diversity. When the river inundation pulse occurred, a reduction in total zooplankton density was observed due to the dilution effect and a significant increase in species richness and diversity was recorded. Lateral water influx from the river to the lacustrine environment acts as a temporary disturbance factor on the zooplankton community structure. Zooplankton species composition presented some modifications between the two periods. Zooplankton organism drift in water from the river to the lake, removal of individuals from the aquatic macrophytes, and eclosion of resting eggs from sediment are probable factors that can increase zooplankton species richness immediately after lateral pulse inundation with water by the river.
\end{abstract}

Keywords: fragmentation, lake, hydrologic connectivity, zooplankton, structure.

\section{Fragmentação temporária de lagoa marginal a um rio e seus efeitos na estrutura e organização da comunidade zooplanctônica}

\section{Resumo}

Uma lagoa lateral a um rio (Lagoa do Coqueiral marginal ao Rio Paranapanema na zona de sua desembocadura na Represa de Jurumirim, São Paulo, Brasil) apresentou segmentação em quatro corpos de água pequenos e isolados entre si quando, durante um período prolongado de seca, ocorreu ruptura da associação entre os dois ambientes. O objetivo deste trabalho foi comparar as modificações temporais na estrutura da comunidade zooplanctônica (abundância total, riqueza e diversidade de espécies) nestes quatro corpos de água. Em dois períodos - um, na fase de isolamento do lago em relação ao rio, e outro, na fase de restauração da conectividade hidrológica - foram efetuadas amostragens do zooplâncton e medidas de fatores abióticos. Um efeito de concentração na abundância do zooplâncton foi registrado com o avançar da seca, contudo sem alterações significativas na riqueza e diversidade de espécies. Com o pulso de inundação pelo curso de água, uma redução na densidade total do zooplâncton ocorreu na lagoa por efeito de diluição e um aumento substancial na riqueza e diversidade específica foi registrado. O influxo lateral de água do rio para o ambiente lacustre atua como fator de distúrbio na estrutura da comunidade zooplanctônica, de caráter temporário. A composição em espécies de zooplancton apresentou modificações na comparação entre os dois períodos. A deriva de organismos zooplanctônicos com a entrada de água do rio na lagoa, a remoção de indivíduos associados às macrófitas aquáticas e a eclosão de ovos de espécies em diapausa presentes no sedimento são os possíveis fatores causadores do aumento de riqueza em espécies zooplanctônicas logo após o pulso de inundação lateral pelo curso de água.

Palavras-chave: fragmentação, lagoa, conectividade hidrológica, zooplancton, estrutura.

\section{Introduction}

Fragmentation of the great biomes is apparently an irreversible process. In The Amazon, deforestation changes tropical rain forest into a mosaic of forested vegetation, where habitat quality for some species is severely affected. Fragments are remnants of natural vegetation surrounded by a variable area (the matrix)

with agricultural characteristics or pasture. In function of their degree of isolation, size, and distance in relation to other fragments, a set of consequences on biota can be detected (Saunders et al., 1991). Habitats become less favourable to species and decrease their aptitudes, and a consequent reduction in species richness is expected 
(Olifiers and Cerqueira, 2006). The degree of fragment isolation can be significantly reduced through "ecological corridors" (Rocha et al., 2006). They consist of surfaces allowing connectivity, i.e, the capacity for organisms, seeds and pollen grains to flow between contiguous fragments.

Many bodies of water can be distinguished in the flood plain. Aquatic systems include main and secondary river channels and lentic ecosystems of various origins (Henry, 2003). Some lakes have temporary or permanent connections with rivers and others remain isolated, with low or close associations with lotic systems depending on their location on the plain and distance from rivers; this is a way to evaluate hydrologic connectivity (Galat et al., 1997). In low water phases, lentic environments can be identified as true "aquatic fragments" in the plain, while in high water periods after inundation pulse, a single large system is seen; this is especially true during the extraordinary floods which occur in the Mato Grosso Pantanal. The hydrometric level rises higher than the river channel overflow leading to lateral hydrologic connectivity (Moulton and Souza, 2006). The lateral inundation water pulse allows an association between lacustrine "fragments" in the plain during the low water phase, and the implanted connection will function as true "ecological corridors" between the lakes where there is notable transfer of particulate substances, dissolved organic matter and biota.

Flood area and hydroperiod duration are important factors in determining the composition and structure of fauna in temporary waters (Sheldon et al., 2002). In Espolla Pond, a characteristically temporary environment in the Banyoles karstic area of the NE Iberian Peninsula, Boix et al. (2001) in a tri-annual study, found a significant relationship between flooded area and macro-invertebrate and micro-crustacean richness but only significant correlation between macro-invertebrate richness and hydroperiod duration. Galindo et al. (1994), in a survey of dune ponds in the Doñana National Park (SW Spain), found high zooplankton species richness and diversity in temporary ponds, especially in those with dense stands of aquatic macrophytes. Species diversity in all the ponds tended to be higher during the inundation period, while total zooplankton abundance was usually low, but with higher values in permanent or semi-permanent ponds.

When a river is dammed to form a reservoir for hydro-electric generation, marginal lakes in the plain completely disappear due to submersion of all neighbouring areas; this was the case in the Paraná River floodplain after the formation of Porto Primavera reservoir, São Paulo - Mato Grosso (Sendacz and Monteiro Jr., 2003). Lateral lentic environments in reservoir tributary mouth zones present a change in the nature of their association with rivers. Lakes change from being isolated or temporarily linked to rivers into environments permanently associated with lotic systems. In this situation, marginal lakes have permanent hydrologic connectivity with riv- ers, and the hydrological pulse action is greatly reduced due to the accumulated water mass behind the downstream dam. This condition was found in lateral lakes of the Paranapanema River mouth zone into Jurumirim Reservoir, São Paulo (Henry, 2005). However, the reservoir operation and drought can lead to a disruption in the hydrologic connectivity between lotic and lateral lentic ecosystems.

This work describes the effects of a prolonged drought and consequent disruption of hydrological connectivity between a river and a lateral lake and its reestablishment on the zooplankton community structure and organization. We intend to show: a) a concentration effect on zooplankton abundance; b) a reduction on species richness and diversity with drought duration and a change in zooplankton composition; c) the lateral pulse inundation of the Paranapanema River acting as a disturbance factor increasing zooplankton community species richness and modifying its structure.

\section{Material and Methods}

\subsection{Study area}

A lateral lake (Coqueiral Lake) of the Paranapanema River, located at the mouth zone into the Jurumirim Reservoir (São Paulo, Brazil) was selected. The lake has a permanent hydrological connection with the river, but with a low area elasticity coefficient despite an annual water level fluctuation of $2.7 \mathrm{~m}$ (Henry et al., 2005). A prolonged drought in 1999 led to a significant level reduction in the Paranapanema River disconnecting the lake and river. This began on October $14^{\text {th }}, 1999$ and continued until December 20 2000 (Henry, 2005). The lake changed from potamophase to limnophase (sensu Neiff, 1999). Afterwards, the river overflowed and the Coqueiral Lake connection was re-established (Figure 1). During the first month of reconnection, daily mean water level increase was $2.9 \mathrm{~cm}$. Water level stability was evident from the end

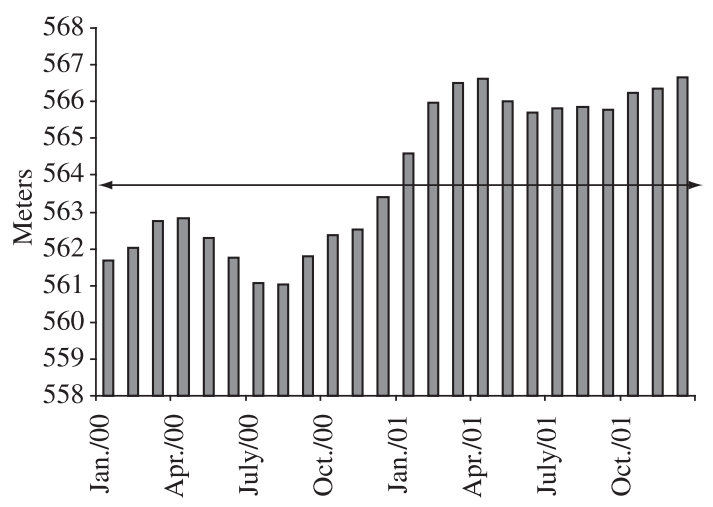

Figure 1. Montly variation on stages $(\mathrm{m})$ of the Paranapanema River (the continuous horizontal line at $563.6 \mathrm{~m}$ water level reproduces the hydrometric level of a frontier between overflow and disconnection of lateral lakes with the river). 
of February 2001 (Figure 1). In function of the topography of Coqueiral Lake bottom (Henry, 2005), the lentic environment was fragmented into some isolated water bodies during the prolonged drought period (Figure 2) due to water loss from evaporation, and surface and hyporheic fluxes. Coqueiral Lake was reduced to around $45 \%$ in November 1999 and 32\% in May 2000 of its original inundated surface area $\left(641,263 \mathrm{~m}^{3}\right)$, measured before the prolonged drought episode (Henry, 2003, 2005). There was, however, an estimated doubling of the area covered by floating Eichhornia azurea (Swartz) Kunth in the first six months of the drought period from $12,000 \mathrm{~m}^{2}$ in November 1999, to 27,509 $\mathrm{m}^{2}$ in May 2000 due to a nitrogen and phosphorus concentration effect in remnant water (Henry, 2003). Coqueiral Lake fragmentation provided an opportunity to study their effect on zooplankton community structure and organization in periods before and after reconnection to the Paranapanema River and compare with data obtained prior to the prolonged drought (Martins and Henry, 2004). Another study during the prolonged drought showed a reduction in Coqueiral Lake phytoplankton taxa number and diversity indices with drought progression (Henry et al., 2006). The algae community was dominated by Cryptophyceae, with Chroomonas spp. and Cryptomonas brasiliensis Castro, C. Bic. and D. Bic. being dominant in May and August 2000, respectively.

\subsection{Sampling methods}

Surface water samples were taken for zooplankton every three months (April, July and October, 2000; February and May, 2001) by bucket from 12 sites in Coqueiral Lake (3 sites each in "fragments" A and C, 4 sites in fragment $\mathrm{B}$, and 2 sites in fragment $\mathrm{D}$, Figure 2). Between 50 and $120 \mathrm{~L}$ of water was filtered in a $50 \mu \mathrm{m}$ mesh net depending on time of year and local depth. Species were identified by keys from Edmondson (1959), Brandlova et al. (1972), Rocha and MatsumuraTundisi (1976), Koste (1978), Reid (1985, 1988), Korovchinsky (1992), and Loureiro (1997). Organism

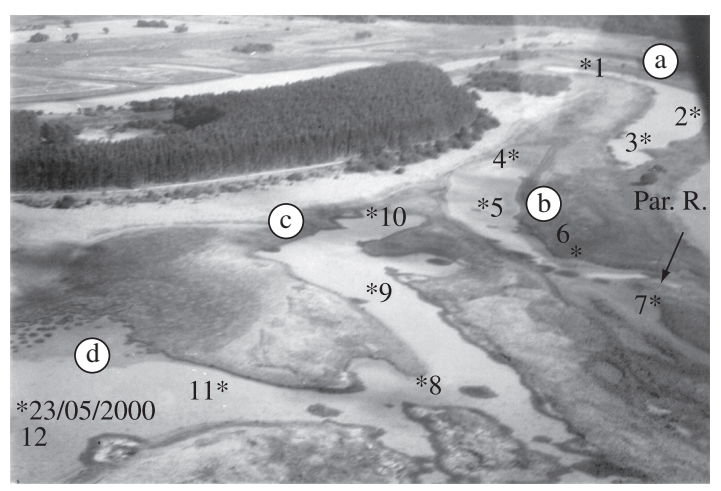

Figure 2. Aerial photography of the four fragments (a to d) of Coqueiral Lake and location of sampling sites in each fragment (the arrow shows the connection site with the Paranapanema (R. Par.). densities (organisms $/ \mathrm{m}^{3}$ ) were assessed by counting $5 \mathrm{~mL}$ sub-samples in an acrylic chamber for micro-crustaceans and copepods under a Zeiss ${ }^{\circledR}$ stereoscopic microscope, and in $1 \mathrm{~mL}$ in Sedegwick-Rafter for rotifers, protozoans and nauplii, under a Zeiss optical microscope. Relative abundance of the main zooplankton groups sampled in each fragment and period is expressed as a percentage of total community density.

Species richness is represented by the mean number of species found in each fragment. Species diversity was computed by the Shannon-Weaver index (Krebs, 1972). The equitability is the ratio between computed and maximum diversity indices (Krebs, 1972). Constancy index for each species was determined with $\mathrm{C}=(\mathrm{n}-100) / \mathrm{N}$ (Dajoz, 1973), where $\mathrm{C}=$ constancy index, $\mathrm{n}=$ number of samples containing the species, and $\mathrm{N}=$ total number of samples. Species in over $50 \%$ of samples were considered constant; in 26 to $50 \%$ as accessory, and in up to $25 \%$ as accidental.

The following abiotic variables were measured in order to show possible variations between fragments and periods: a) surface water temperature, with a Toho Dentam ET-3 thermistor; b) sampling site depth by manual sounding; c) water transparency, with a Secchi disk; d) dissolved oxygen in water, by the Winkler method (Golterman and Clymo, 1969); e) water $\mathrm{pH}$, with a Micronal B-380 pHmeter; f) water conductivity corrected to $25{ }^{\circ} \mathrm{C}$, with a Hatch Model conductivity 2511 metre; g) total alkalinity by titration with $0.01 \mathrm{~N}$ $\mathrm{H}_{2} \mathrm{SO}_{4}$ (McKareth et al., 1978); and h) chlorophyll-a by water filtration in Millipore AP4004700 filters with pigment extraction in $90 \%$ acetone (Golterman and Clymo, 1969).

\subsection{Statistical analysis}

Possible similarities between environmental variables from fragments and sampling periods were tested by ANOVA, and significant differences calculated using the Tukey test and SAS system software. The normality and homeostacity test for physical and chemical water data was not necessary since variation coefficients $(\mathrm{CV})$ and determination $\left(\mathrm{R}^{2}\right)$ values were acceptable (Pião, personal communication). Fragment comparisons were by similarity analysis using the UPGMA methods (Valentin, 2000), with species diversity in each fragment being the descriptor variable. Pearson correlation analysis was used to assess the influence of abiotic variables on zooplankton community (richness and density).

\section{Results}

Except for water temperature, the other abiotic factors presented significant spatial and temporal variations (Table 1). According to temporal analysis, the lowest water temperatures were in July (Table 1). The lowest levels in Coqueiral Lake were recorded in the drought period (April, July and October), with depths significantly increasing after reconnection to the Paranapanema River 
Nadai, R. and Henry, R.

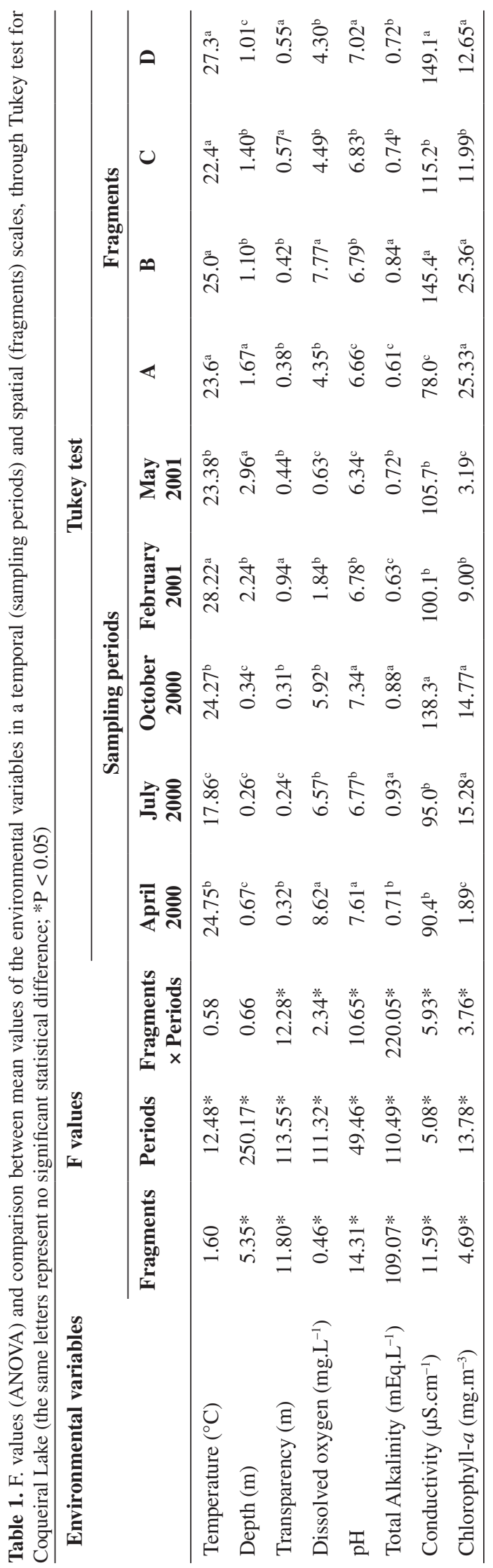


(February and May; Table 1). Low water transparencies were observed during the isolation period (April, July and October). After reconnection, high transparency in February was due to a dilution effect, and the reduction in Secchi disk readings in May were probably due to resuspension of bottom material (Table 1). Dissolved oxygen concentrations and water $\mathrm{pH}$ decreased in February and May, after reconnection, compared with values during isolation. Total water alkalinity was lower in the second month (February) after reconnection; however, values in May were similar to those from the three isolation periods (Table 1). Water conductivity and chlorophyll-a presented an increasing trend in the three lake isolation periods, and decreased after reconnection to the river (Table 1).

Spatially (comparison between fragments), the highest oxygen, total alkalinity, and chlorophyll-a values were found in the fragment $\mathrm{B}$ site nearest to the river connection (Table 1). In the fragment A site most isolated from the river, lowest water transparency, $\mathrm{pH}$, conductivity, alkalinity and highest depth were recorded (Table 1). In the fragment D site farthest from connection to the river, the lowest depth, dissolved oxygen, and chlorophyll- $a$, and the highest water $\mathrm{pH}$ and conductivity were observed (Table 1).

During the study period, 110 taxa were recorded in the zooplankton community, distributed in Cladocera (29 taxons), Copepoda (10 taxons), Rotifera (54 taxons) and Protozoa (17 taxons). Table 2 shows each taxon occurrence and constancy indices during the year in each fragment site. The only taxon considered constant $(>50 \%$ presence in all samples) during the study in all 5 periods was Polyarthra sp. (83\%). Conochilus coenobasis, C. unicornis and Notodiaptomus spp. were constant in 4 periods (Table 2). Difflugia spp. and Trichodina spp. were constant in 3 periods. Epiphanes sp., Keratella cochlearis, Mytilinea sp., Thermocyclops decipiens, Ceriodaphnia cornuta Rigaud, C. cornuta, C. cornuta cornuta, Diaphanosoma birgei, Moina minuta and an unidentified ciliate were constant in two periods. Finally, Arcella conica, Difflugia corona, Brachionus dolobrastus, B. falacatus, Conochilus natans, Filinia longiseta. F. terminalis, Gastropus sp., Hexarthra sp., Lecane bulla, L. curvicornis, Plationus patalus, cf. Sinantherina, Eucyclops ensifer, Ceriodaphnia silvestrii, Diaphanosoma brevirreme, and Moina cf. reticulate were constant in one of the 5 sample periods (Table 2). All other taxa presented constancy indexes $<50 \%$ (Table 2). A decrease in number of constant species was recorded with reduced fragment area and volume. In April 2000, 14 constant taxa were found, followed by 8 in July, and 7 in October. In February 2001, about two months after reconnection of all Coqueiral Lake fragments by water inundation from the Paranapanema River, the number of constant species increased to 21 taxa. Three months later, in May 2001, the number decreased to 11 constant species.
In April 2000, mean zooplankton species richness did not vary in 4 Coqueiral Lake fragments (Table 3 ). In July, fragment $\mathrm{A}$ and $\mathrm{C}$ richness displayed less variation than in the previous sampling period (Table 3). However, significant reductions were seen for fragments B (sites 4 and 7) and D (sites 11 and 12) due to sediment exposition. In October, the lowest mean zooplankton richness was found in fragment B (Table 3). In February, two months after reconnection, the highest zooplankton richness values were recorded in all fragments. May's mean species richness values in the 4 fragments were approximately similar to those from April of the previous year (Table 3). The highest temporal variability in mean zooplankton species richness was found in fragment $\mathrm{B}(\mathrm{CV}=69 \%)$, followed by fragment $\mathrm{D}(\mathrm{CV}=57 \%), \mathrm{C}$ $(\mathrm{CV}=34 \%)$, and $\mathrm{A}(\mathrm{CV}=29 \%)$.

Mean zooplankton density in the 4 fragments (sampling site means) ranged from 204,000 (May 2001, fragment D) to 2,074,000 organisms.m ${ }^{-3}$ (October 2000, fragment C; Figure 3). Sediment exposure in fragment $\mathrm{D}$ occurred in two sampling sites in July, and in fragment B in one and two sites in July and October, respectively; in these cases, the means were calculated with fewer repetitions than the others periods. The highest annual variability was recorded in fragment $\mathrm{B}(\mathrm{CV}=$ $97 \%$; mean $=607,000 \pm 587,000$ individuals. $\mathrm{m}^{-3}$ ), followed by fragment $\mathrm{D}(\mathrm{CV}=77 \%$; mean $=596,000 \pm$ 456,000 individuals. $\left.\mathrm{m}^{-3}\right)$, fragment $\mathrm{C}(\mathrm{CV}=76 \%$; $875,000 \pm 667,000$ individuals. $\mathrm{m}^{-3}$ ) and fragment $\mathrm{A}\left(\mathrm{CV}=51 \% ; 543,000 \pm 274,000\right.$ individuals. $\left.\mathrm{m}^{-3}\right)$. Considering the Coqueiral Lake as a single body of water (4 fragments, 12 sampling sites), the highest mean zooplankton density was recorded in October $(1,494,000 \pm$ 492,000 individuals. $\mathrm{m}^{-3}$; $\mathrm{CV}=34 \%$ ), followed by July $\left(763,000 \pm 318,000\right.$ individuals.m $\left.{ }^{-3} ; \mathrm{CV}=42 \%\right)$, April, $2000\left(544,000 \pm 187,000\right.$ individuals. $\left.\mathrm{m}^{-3} ; \mathrm{CV}=34 \%\right)$, February $\left(363,000 \pm 177,000\right.$ individuals. $\left.\mathrm{m}^{-3} ; \mathrm{CV}=34 \%\right)$, and May $2001\left(180,000 \pm 55,000\right.$ individuals. $\mathrm{m}^{-3}$; CV = $31 \%$ ). In relation to relative density, the predominant organism group was Rotifera (11 out of 20 means; Figure 3); the second was Copepoda (five occurrences). Protozoa attained high relative densities in July and October in fragment B and, in October in fragment C (Figure 3).

Except for fragment A, species diversity indices and equitabilities were higher in February, 2001 (Figure 4). The highest values in fragment A were recorded in April 2000. Annual species diversity index variability was higher in fragment $\mathrm{B}(\mathrm{CV}=35 \%)$ than fragments $\mathrm{C}(\mathrm{CV}=21 \%), \mathrm{D}(\mathrm{CV}=17 \%)$, and $\mathrm{C}$ (7\%).

Species richness had significant positive correlation with water temperature and transparency in all fragments (Table 4). Negative and positive correlations with dissolved oxygen concentrations and depth, respectively, were only recorded in fragments $\mathrm{A}$ and $\mathrm{B}$. Water $\mathrm{pH}$ and conductivity had positive correlations with species richness in fragment D. Total zooplankton abundance negatively correlated with most environmental variables 
Table 2. Ocurrence of zooplanktonic taxa in each site (1 to 12) and fragment (A to D) of Coqueiral Lake in April (1), July (2) and October (3) 2000 and February (4) and May (5) 2001, and respective constancy indexes (CI to CI ; $_{5}$ TCI: for all the five sampling periods). In bold and grey constancy indexes ( $>50 \%$ and $>26 \%$ and $<50 \%$, respectively) for constant and accessory species.

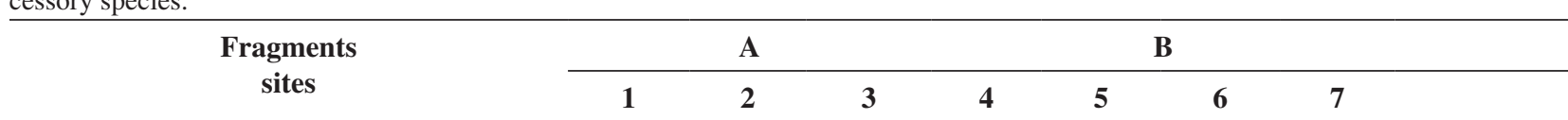

Protozoa

Arcella conica (Playfair, 1917)

Arcella costata (Ehrenberg, 1847)

Arcella dentata (Ehrenberg, 1838)

Arcella discoides (Ehrenberg, 1843)

Arcella hemisphaerica (Perty, 1852)

Arcella vulgaris (Ehrenberg, 1830)

Centropyxis sp.

Unidentified ciliate

Diffugia corona (Wallich, 1864)

Difflugia gramen (Pénard, 1902)

Diffugia lobostoma

Diffugia spp.

Lesquereusia sp.

Nebella sp.

Tintinopsis spp.

Trichodina spp.

Vorticella spp.

Rotifera

Ascomorpha sp. Perty, 1850

Asplanchna sp. Goss, 1850

Anuraeopsis sp. Lauterborn, 1900

Bdelloidea

Brachionus angularis Gosse, 1851

Brachionus dolobratus Haring, 1915

Brachionus falcatus Zacharias, 1898

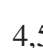

$\begin{array}{lllllll}4,5 & 4,5 & 4,5 & 4,5 & 4 & 3,4 & 1,4 \\ 4 & 4 & 4 & - & - & 4 & - \\ 2 & - & - & - & - & - & - \\ 5 & - & 4 & - & - & 5 & - \\ 4 & - & - & - & - & - & - \\ - & - & - & - & - & 3 & 1 \\ - & - & 1 & 1,4 & - & 3 & - \\ 4 & 4,5 & 1,4,5 & 4,5 & 3,5 & 1,3,5 & \\ 5 & 4,5 & 4 & 4 & 4 & 4,5 & 4 \\ - & 4 & 4 & 4 & 4 & 5 & - \\ - & - & 4 & - & - & - & -\end{array}$

Brachionus forficula Wierzejski, 1891

$1,4,5 \quad 1,4 \quad 1,4,5$

$1,4,5 \quad 1,2,4,5 \quad 1,4,5$

4,5

- $\quad$ - 4

1,4

-

4

1

Brachionus mirus Daday, 1905

Brachionus quadridentatus Hermann, 17835

Cephallodela sp. Bory de St. Vincent, 18265

Collotheca sp. Harring, 1913

Conochilus coenobasis (Skorikov, 1914)

Conochilus natans (Seligo, 1900)

$1,2,3,4 \quad 1,2,3,4 \quad 1,2,3,4 \quad 1,4 \quad 1,3,4,5 \quad 1,3,4,5 \quad 4$

Conochilus unicornis Rousselet, 1892

34

Colurella sp. Bory de St. Vincent, 1824

$4 \quad 4$

$1,2,3,4 \quad 1,2,3 \quad 1,2,3,4$

$4 \quad 4 \quad 4$

Euchlanis sp. Ehrenberg, 1832 


\begin{tabular}{|c|c|c|c|c|c|c|c|c|c|c|}
\hline \multicolumn{3}{|c|}{ C } & \multicolumn{2}{|c|}{ D } & \multicolumn{6}{|c|}{ Constancy indexes } \\
\hline 8 & 9 & 10 & 11 & 12 & $\mathrm{CI}_{1}$ & $\mathrm{CI}_{2}$ & $\mathrm{CI}_{3}$ & $\mathrm{CI}_{4}$ & $\mathbf{C I}_{5}$ & TCI \\
\hline- & $2,4,5$ & 4,5 & 4 & 3 & 8 & 14 & 17 & 83 & 42 & 38 \\
\hline- & 2 & - & - & - & 0 & 14 & 0 & 42 & 0 & 9 \\
\hline- & - & - & - & - & 0 & 14 & 0 & 0 & 0 & 2 \\
\hline- & - & - & 4 & - & 0 & 0 & 0 & 0 & 17 & 8 \\
\hline- & - & - & - & - & 0 & 0 & 0 & 8 & 0 & 2 \\
\hline- & - & - & - & - & 17 & 0 & 8 & 0 & 0 & 4 \\
\hline- & 2,3 & 3,4 & 4 & 3 & 17 & 14 & 33 & 25 & 0 & 19 \\
\hline $1,4,5$ & $2,3,4,5$ & $3,4,5$ & 4,5 & 3,5 & 25 & 14 & 42 & 67 & 83 & 45 \\
\hline- & - & 3,4 & 4 & 4 & 0 & 0 & 8 & 75 & 25 & 25 \\
\hline- & - & - & 4 & - & 0 & 0 & 0 & 42 & 8 & 11 \\
\hline- & - & - & - & - & 0 & 0 & 0 & 8 & 0 & 4 \\
\hline $1,2,4$ & $2,4,5$ & $3,4,5$ & 1,4 & 4,5 & 67 & 43 & 8 & 100 & 75 & 62 \\
\hline 3 & 2,3 & 3,4 & 4 & 3 & 17 & 29 & 42 & 33 & 0 & 25 \\
\hline- & - & 3 & - & 3 & 0 & 0 & 25 & 42 & 0 & 15 \\
\hline- & - & - & 3 & 1 & 33 & 0 & 8 & 17 & 0 & 13 \\
\hline $1,2,3,4$ & $1,3,4$ & $1,3,4$ & $1,3,4$ & $1,3,4$ & 100 & 14 & 75 & 58 & 8 & 57 \\
\hline 1 & 4 & - & 4 & 3 & 17 & 0 & 8 & 42 & 8 & 17 \\
\hline- & 4,5 & - & 5 & - & 0 & 0 & 8 & 17 & 17 & 9 \\
\hline- & 4,3 & 4,3 & 4,5 & 4,3 & 0 & 0 & 25 & 50 & 17 & 23 \\
\hline & - & 3 & 3 & - & 0 & 0 & 25 & 0 & 0 & 8 \\
\hline- & - & 4 & 4 & - & 0 & 0 & 0 & 25 & 25 & 11 \\
\hline- & - & - & - & - & 0 & 0 & 0 & 0 & 8 & 4 \\
\hline 1,4 & - & - & 1 & - & 58 & 14 & 0 & 25 & 0 & 21 \\
\hline 5 & 1 & 1,4 & 1 & 4 & 83 & 43 & 0 & 50 & 17 & 40 \\
\hline & - & - & - & 1 & 42 & 29 & 0 & 25 & 0 & 19 \\
\hline- & - & - & - & 4 & 0 & 0 & 0 & 8 & 0 & 2 \\
\hline- & - & 1 & - & 3 & 8 & 0 & 8 & 0 & 25 & 9 \\
\hline- & - & - & - & - & 0 & 0 & 0 & 0 & 8 & 2 \\
\hline- & - & - & - & - & 0 & 0 & 0 & 8 & 0 & 2 \\
\hline 1 & 1,3 & $1,3,4$ & $1,2,3,4$ & 5 & 83 & 57 & 58 & 75 & 25 & 64 \\
\hline 2,4 & - & 4,5 & $3,4,5$ & 3,4 & 0 & 14 & 25 & 83 & 17 & 30 \\
\hline $1,2,3,4$ & $1,3,4$ & $1,3,4$ & $1,3,4$ & $1,3,4$ & 100 & 57 & 83 & 83 & 8 & 70 \\
\hline- & - & - & - & - & 0 & 0 & 0 & 8 & 0 & 2 \\
\hline- & - & - & - & - & 0 & 0 & 0 & 8 & 0 & 2 \\
\hline
\end{tabular}


Table 2. Continued...

\begin{tabular}{|c|c|c|c|c|c|c|c|}
\hline \multirow{2}{*}{$\begin{array}{l}\text { Fragments } \\
\text { sites }\end{array}$} & \multicolumn{3}{|c|}{$\mathbf{A}$} & \multicolumn{4}{|c|}{ B } \\
\hline & 1 & 2 & 3 & 4 & 5 & 6 & 7 \\
\hline Epiphanes sp. Ehrenber, 1832 & 4,5 & 4,5 & 4,5 & 4,5 & 3,5 & 5 & 5 \\
\hline Filinia terminalis (Plate, 1886) & 5 & 5 & 5 & 5 & 5 & 5 & 5 \\
\hline Gastropus sp. Imhof, 1898 & 2,4 & 2 & $2,3,4$ & 4 & 4 & 4 & 4 \\
\hline Hexarthra sp. Schmarda, 1854 & $1,2,3$ & $1,2,3$ & $1,2,3$ & 1 & 1,4 & $1,3,4$ & 4 \\
\hline Keratella americana Carlin, 1943 & 2 & 2 & 2 & - & - & - & - \\
\hline Keratella cochlearis (Gosse, 1851) & $1,2,3,5$ & 2,3 & $1,2,3$ & 1 & 3 & 3 & \\
\hline Keratella lenzi Hauer, 1953 & - & - & 4 & 4 & 4 & 4,5 & - \\
\hline Keratella tropica (Apsten, 1907) & - & - & - & - & 3 & 3 & - \\
\hline Lacinularia sp. Schweigger, 1826 & - & - & - & - & - & - & - \\
\hline Lecane bulla (Gosse, 1851) & 5 & 4 & 5 & 4,5 & - & 3,5 & 5 \\
\hline Lecane curvicornis (Murray, 1903) & 4,5 & 4,5 & 4,5 & 4,5 & 4 & 3,4 & 4 \\
\hline Lecane decipiens (Murray, 1903) & - & 4 & - & - & - & - & - \\
\hline Lecane cf. furcata (Stokes, 1896) & - & - & - & - & - & - & 5 \\
\hline Lecane hamata (Stokes, 1896) & 5 & 5 & - & - & - & - & - \\
\hline Lecane leontina (Turner, 1892) & - & - & - & - & - & - & - \\
\hline Lecane papuanatata (Murray, 1913) & 5 & - & - & 5 & - & 1 & - \\
\hline Lecane quadridentata (Ehrenberg, 1832) & - & - & - & - & - & 3 & - \\
\hline Lecane sp. Nitzsch, 1827 & 5 & - & 4 & 5 & - & - & - \\
\hline Lepadella sp. Bory de St. Vincent, 1826 & 4 & 4 & 1,4 & 1 & - & - & - \\
\hline Lepadella patella (Muller, 1786) & - & - & - & 4 & - & - & - \\
\hline Macrochaetus sp. Perty, 1850 & - & 4 & - & 4 & - & - & - \\
\hline Mytilina sp. Bory de St. Vincent, 1826 & 4,5 & 4,5 & 5 & 4,5 & 3,4 & 1,4 & 4,5 \\
\hline Notommata sp. Ehrenber, 1830 & - & 4 & - & 4 & - & - & - \\
\hline Plationus patulus (Muller, 1786) & 2,4 & - & - & 4 & $1,3,4$ & $1,3,4,5$ & 1,4 \\
\hline Platyias sp. Harring, 1913 & - & - & - & 4 & 4 & 4 & 4 \\
\hline Polyarthra sp. Ehrenberg, 1834 & $2,3,4,5$ & $2,3,4,5$ & $1,2,3,4,5$ & $1,4,5$ & $3,4,5$ & $1,3,4,5$ & $1,4,5$ \\
\hline Pompholix sp. Gosse, 1851 & 2 & 1 & 1 & 1 & 2 & - & - \\
\hline Ptygura sp. Ehrenberg, 1832 & 2 & 2 & 2 & - & - & - & - \\
\hline Scaridium sp. Ehernberg, 1830 & - & 4 & - & - & - & - & - \\
\hline $\begin{array}{l}\text { cf. Sinantherina sp. } \\
\text { Bory de St. Vincent, } 1826\end{array}$ & 4,5 & 4 & - & - & 4,5 & 4 & 4 \\
\hline Synchaeta sp. Ehrenberg, 1832 & 2 & 2,4 & 2,3 & - & - & - & - \\
\hline Testudinella sp. Bory de St. Vincent, 1826 & - & 4 & 4 & - & 4,5 & 4 & 4 \\
\hline Trichocerca bicristata (Gosse, 1887) & - & - & - & - & 4 & 4 & 4 \\
\hline $\begin{array}{l}\text { Trichocerca capucina } \\
\text { (Wierzejski and Zacharias, 1893) }\end{array}$ & - & 3 & - & - & - & - & - \\
\hline Trichocerca similis (Wierzejski, 1893) & - & - & - & - & 5 & 5 & 5 \\
\hline Trichocerca sp. Lamarck, 1801 & 5 & 4 & - & 5 & - & 3 & - \\
\hline \multicolumn{8}{|l|}{ Copepoda } \\
\hline Eucyclops ensifer & - & 3,4 & 2,4 & 4 & 3,4 & 2,4 & 1,4 \\
\hline
\end{tabular}




\begin{tabular}{|c|c|c|c|c|c|c|c|c|c|c|}
\hline \multicolumn{3}{|c|}{$\mathbf{C}$} & \multicolumn{2}{|c|}{ D } & \multicolumn{6}{|c|}{ Constancy indexes } \\
\hline 8 & 9 & 10 & 11 & 12 & $\mathrm{CI}_{1}$ & $\mathrm{CI}_{2}$ & $\mathrm{CI}_{3}$ & $\mathrm{CI}_{4}$ & $\mathrm{CI}_{5}$ & TCI \\
\hline 4,5 & 4,5 & 4 & 4,5 & 4 & 0 & 0 & 8 & 75 & 83 & 38 \\
\hline 5 & 5 & 5 & 5 & 5 & 0 & 0 & 0 & 0 & 100 & 23 \\
\hline 4 & - & 3 & - & - & 0 & 43 & 17 & 58 & 0 & 23 \\
\hline 1 & - & $1,3,4$ & 1 & 1 & 100 & 43 & 42 & 25 & 0 & 36 \\
\hline- & - & - & - & - & 0 & 43 & 0 & 0 & 0 & 6 \\
\hline 1,2 & $1,2,3$ & - & 3 & - & 42 & 71 & 58 & 0 & 0 & 34 \\
\hline 4 & 4 & 3,4 & - & - & 0 & 0 & 8 & 50 & 8 & 19 \\
\hline- & - & - & - & 3 & 0 & 0 & 25 & 0 & 0 & 6 \\
\hline- & - & 4 & - & - & 0 & 0 & 0 & 8 & 0 & 2 \\
\hline- & 5 & $3,4,5$ & 3 & 3,5 & 0 & 0 & 33 & 25 & 67 & 28 \\
\hline 4 & 5 & 3,4 & - & - & 0 & 0 & 17 & 75 & 42 & 30 \\
\hline- & - & - & - & - & 0 & 0 & 0 & 8 & 0 & 2 \\
\hline- & - & - & - & - & 0 & 0 & 0 & 0 & 8 & 2 \\
\hline- & - & - & - & - & 0 & 0 & 0 & 0 & 17 & 4 \\
\hline- & - & - & 4 & - & 0 & 0 & 0 & 8 & 0 & 2 \\
\hline 1 & - & - & 3 & 3 & 17 & 0 & 17 & 0 & 17 & 11 \\
\hline- & - & - & - & - & 0 & 0 & 8 & 0 & 0 & 2 \\
\hline- & - & - & - & - & 0 & 0 & 0 & 8 & 17 & 6 \\
\hline- & 4 & 3,4 & - & - & 17 & 0 & 8 & 42 & 0 & 15 \\
\hline- & - & - & - & - & 0 & 0 & 0 & 8 & 0 & 2 \\
\hline- & - & - & - & - & 0 & 0 & 0 & 17 & 0 & 4 \\
\hline 4 & 4,5 & 3,5 & 4,5 & 5 & 8 & 0 & 17 & 75 & 75 & 40 \\
\hline- & - & - & - & - & 0 & 0 & 0 & 17 & 0 & 4 \\
\hline 1,3 & 2,3 & 3 & 3 & 3 & 33 & 29 & 58 & 42 & 0 & 36 \\
\hline- & - & 3 & 5 & - & 0 & 0 & 8 & 33 & 8 & 13 \\
\hline $1,3,5$ & $1,2,3,4,5$ & $1,3,5$ & $3,4,5$ & $1,3,4,5$ & 100 & 57 & 83 & 83 & 100 & 83 \\
\hline- & 1,2 & 1 & - & - & 42 & 43 & 0 & 0 & 0 & 15 \\
\hline- & 4 & - & 4 & - & 0 & 43 & 8 & 17 & 0 & 9 \\
\hline- & - & - & - & - & 0 & 0 & 0 & 8 & 0 & 2 \\
\hline- & 4 & 3,4 & 4 & 4 & 0 & 0 & 8 & 75 & 17 & 23 \\
\hline- & 1 & 1 & 5 & 1 & 25 & 43 & 8 & 8 & 8 & 17 \\
\hline- & - & - & 4 & - & 0 & 0 & 8 & 50 & 8 & 15 \\
\hline- & - & 4 & - & - & 0 & 0 & 0 & 33 & 0 & 8 \\
\hline- & 2 & - & - & - & 0 & 14 & 8 & 0 & 0 & 4 \\
\hline 5 & 5 & - & - & - & 0 & 0 & 0 & 0 & 42 & 9 \\
\hline- & - & - & - & 3 & 0 & 0 & 17 & 8 & 17 & 9 \\
\hline- & 4 & 4 & - & 4 & 0 & 29 & 17 & 75 & 0 & 26 \\
\hline
\end{tabular}


Table 2. Continued...

\begin{tabular}{|c|c|c|c|c|c|c|c|}
\hline \multirow{2}{*}{$\begin{array}{l}\text { Fragments } \\
\text { sites }\end{array}$} & \multicolumn{3}{|c|}{$\mathbf{A}$} & \multicolumn{4}{|c|}{ B } \\
\hline & 1 & 2 & 3 & 4 & 5 & 6 & 7 \\
\hline Mesocyclops ogunnus & 4 & - & 1 & - & - & - & - \\
\hline Metacyclops sp. & 3 & 2,4 & - & - & 3 & - & 1 \\
\hline Microcyclops anceps (Richard, 1897) & - & - & - & - & - & - & 1 \\
\hline Notodiaptomus spinuliferus Dussart, 1985 & - & - & 3 & - & 3 & - & - \\
\hline Notodiaptomus spp. & 1,3 & $1,3,4$ & 1,4 & $1,4,5$ & $1,3,4,5$ & $1,4,5$ & $1,4,5$ \\
\hline Paracyclops sp. & - & - & - & - & 1 & - & - \\
\hline Thermocyclops decipiens (Kiefer, 1929) & $1,2,3,4,5$ & 2,4 & $2,3,4,5$ & 4 & 4,5 & 4,5 & 4,5 \\
\hline \multicolumn{8}{|l|}{ Cladocera } \\
\hline Alona pulchella King, 1853 & - & - & - & - & - & 5 & - \\
\hline Alona rectangula Sars, 1861 & - & - & - & - & - & - & - \\
\hline Alona sp. Baird, 1843 & - & - & - & - & 4 & 4,5 & 1,4 \\
\hline cf. Alonella sp. Sars, 1862 & - & - & - & - & - & - & - \\
\hline Bosmina hagmanni Stingelin, 1904 & 3 & 3 & 3 & - & 3 & - & - \\
\hline Bosmia longirostris (O.F.Muller, 1785) & 3 & 3 & 3 & - & 3 & - & - \\
\hline Bosminopsis deitersio Richard, 1895 & - & - & 3 & - & - & - & - \\
\hline Ceriodaphnia cornuta Sars, 1901 & 2,3 & $1,2,3$ & $1,2,3,4$ & 1 & 2,3 & 1 & 1 \\
\hline Ceriodaphnia cornuta intermedia & $1,2,4$ & 1,2 & $1,2,3,4$ & 1,4 & 1 & - & - \\
\hline Ceriodaphnia cornuta rigaudi Richard, 1886 & 1,2 & 1,2 & 1,2 & 1,4 & 1,2 & 1 & 1 \\
\hline Ceriodaphnia silvestrii Daday, 1902 & 3,4 & - & - & 4 & 4 & 4 & 4 \\
\hline Chydorus pubscens Sars, 1901 & - & - & - & - & 3,4 & 4 & 4 \\
\hline Chydorus sp. Leach, 1816 & - & 2 & 4 & - & 3 & - & - \\
\hline Daphnia gessneri Herbst, 1967 & - & - & - & - & - & - & - \\
\hline Diaphanosoma brevireme Sars, 1901 & 4 & 1 & 3,4 & 4 & 4 & 4 & 4,5 \\
\hline Diaphanosoma birgei Korinek, 1981 & 1,3 & 1,3 & $1,3,4$ & 1,4 & $1,3,4$ & $1,4,5$ & $1,4,5$ \\
\hline Diaphanosoma spinulosum Herbst, 1967 & 4 & 4 & 4 & - & - & - & - \\
\hline Euryalona orientalis (Daday, 1898) & - & - & 4 & - & - & - & - \\
\hline Ilyocryptus spinifer Herrick, 1882 & - & - & - & 4 & 4 & 4 & 4 \\
\hline Kurzia longispina (Daday, 1898) & - & - & - & 4 & 2 & - & - \\
\hline Kurzia cf. polispina Hudec, 2000 & - & - & - & - & - & - & - \\
\hline Leydigia cf. propinqua Sars, 1903 & - & - & - & - & 2 & - & - \\
\hline Leydigiopsis brevirostris & - & - & - & - & - & - & - \\
\hline Macrothrix spinosa King, 1853 & - & - & - & 4 & 4 & 4 & 4 \\
\hline Macrothrix superaculeata (Smirnov, 1992) & - & - & - & 1 & 1 & - & 1 \\
\hline Moina micrura Kurz, 1874 & $1,2,3,4,5$ & 1,4 & $1,2,4,5$ & 1 & - & 1 & 1 \\
\hline Moina minuta Hansen, 1899 & $3,4,5$ & $3,4,5$ & 4,5 & 4,5 & $2,3,4,5$ & 4,5 & 4,5 \\
\hline Moina cf. reticulata (Daday, 1905) & 2,5 & 4,2 & 2 & - & - & - & - \\
\hline Simocephalus sp. Schoedler, 1858 & - & 2 & 2 & - & - & - & - \\
\hline
\end{tabular}




\begin{tabular}{|c|c|c|c|c|c|c|c|c|c|c|}
\hline \multicolumn{3}{|c|}{$\mathbf{C}$} & \multicolumn{2}{|c|}{ D } & \multicolumn{6}{|c|}{ Constancy indexes } \\
\hline 8 & 9 & 10 & 11 & 12 & $\mathrm{CI}_{1}$ & $\mathrm{CI}_{2}$ & $\mathrm{CI}_{3}$ & $\mathrm{CI}_{4}$ & $\mathrm{CI}_{5}$ & TCI \\
\hline 4 & 2,4 & 4 & 4 & 4 & 8 & 14 & 0 & 50 & 0 & 15 \\
\hline 2 & - & - & 3 & - & 8 & 29 & 25 & 8 & 0 & 11 \\
\hline- & - & - & - & - & 8 & 0 & 0 & 0 & 0 & 2 \\
\hline- & 3 & - & - & - & 0 & 0 & 25 & 0 & 0 & 6 \\
\hline $1,3,4,5$ & $1,3,4,5$ & $1,3,4,5$ & $1,3,4,5$ & $1,3,4,5$ & 100 & 0 & 80 & 91 & 75 & 75 \\
\hline- & - & - & - & - & 8 & 0 & 0 & 0 & 0 & 2 \\
\hline $1,3,4,5$ & $1,2,3,4,5$ & $3,4,5$ & $1,4,5$ & $1,3,4,5$ & 42 & 43 & 50 & 100 & 83 & 71 \\
\hline
\end{tabular}

\begin{tabular}{|c|c|c|c|c|c|c|c|c|c|c|}
\hline 2 & - & - & 3 & - & 0 & 14 & 8 & 0 & 8 & 6 \\
\hline - & - & - & - & 3 & 0 & 0 & 8 & 0 & 0 & 2 \\
\hline - & - & - & 4 & - & 8 & 0 & 0 & 33 & 8 & 11 \\
\hline- & - & - & - & 3 & 0 & 0 & 8 & 0 & 0 & 2 \\
\hline- & - & 3 & - & - & 0 & 0 & 42 & 0 & 0 & 9 \\
\hline- & - & - & - & - & 0 & 0 & 33 & 0 & 0 & 8 \\
\hline- & - & - & - & - & 0 & 0 & 8 & 0 & 0 & 2 \\
\hline 1,2 & 1 & 1 & 1 & 1,4 & 83 & 71 & 33 & 17 & 0 & 40 \\
\hline $1,2,4$ & 1,2 & 1 & 1,4 & 1 & 83 & 71 & 8 & 42 & 0 & 40 \\
\hline 1,4 & 1,4 & 1,4 & 4 & 1,4 & 92 & 57 & 0 & 50 & 0 & 40 \\
\hline $2,3,4$ & 2,4 & 4 & 3,4 & 4 & 0 & 29 & 25 & 83 & 0 & 28 \\
\hline 2 & - & 4 & - & 3 & 0 & 14 & 17 & 33 & 0 & 13 \\
\hline - & - & - & 3 & - & 0 & 14 & 17 & 8 & 0 & 8 \\
\hline - & - & 3,4 & 1 & 1 & 17 & 0 & 8 & 8 & 0 & 8 \\
\hline 4,5 & 4 & 4 & 4 & 4 & 8 & 14 & 8 & 92 & 0 & 30 \\
\hline $1,2,3,4$ & $1,2,4$ & $1,3,4$ & 1,4 & 1,4 & 100 & 14 & 50 & 83 & 0 & 60 \\
\hline 4 & 4 & - & - & - & 0 & 0 & 0 & 42 & 0 & 9 \\
\hline - & - & - & - & - & 0 & 0 & 0 & 8 & 0 & 2 \\
\hline - & - & - & - & - & 0 & 0 & 0 & 33 & 0 & 8 \\
\hline- & - & - & - & - & 0 & 14 & 0 & 8 & 0 & 4 \\
\hline- & - & - & - & 3 & 0 & 0 & 8 & 0 & 0 & 2 \\
\hline- & - & - & - & - & 0 & 14 & 0 & 0 & 0 & 2 \\
\hline 4 & - & - & - & - & 0 & 0 & 0 & 8 & 0 & 2 \\
\hline 2 & 1,2 & 3,4 & 3,4 & 3 & 8 & 29 & 25 & 50 & 0 & 23 \\
\hline- & - & - & - & - & 25 & 0 & 0 & 0 & 0 & 6 \\
\hline 1,2 & 1,4 & $1,4,5$ & 1,3 & 1 & 92 & 43 & 17 & 42 & 25 & 45 \\
\hline $3,4,5$ & $3,4,5$ & $3,4,5$ & 5 & 5 & 0 & 14 & 50 & 83 & 100 & 55 \\
\hline 2 & - & - & 3 & - & 0 & 57 & 8 & 8 & 8 & 13 \\
\hline 2 & - & - & 3 & - & 0 & 43 & 8 & 0 & 0 & 8 \\
\hline
\end{tabular}




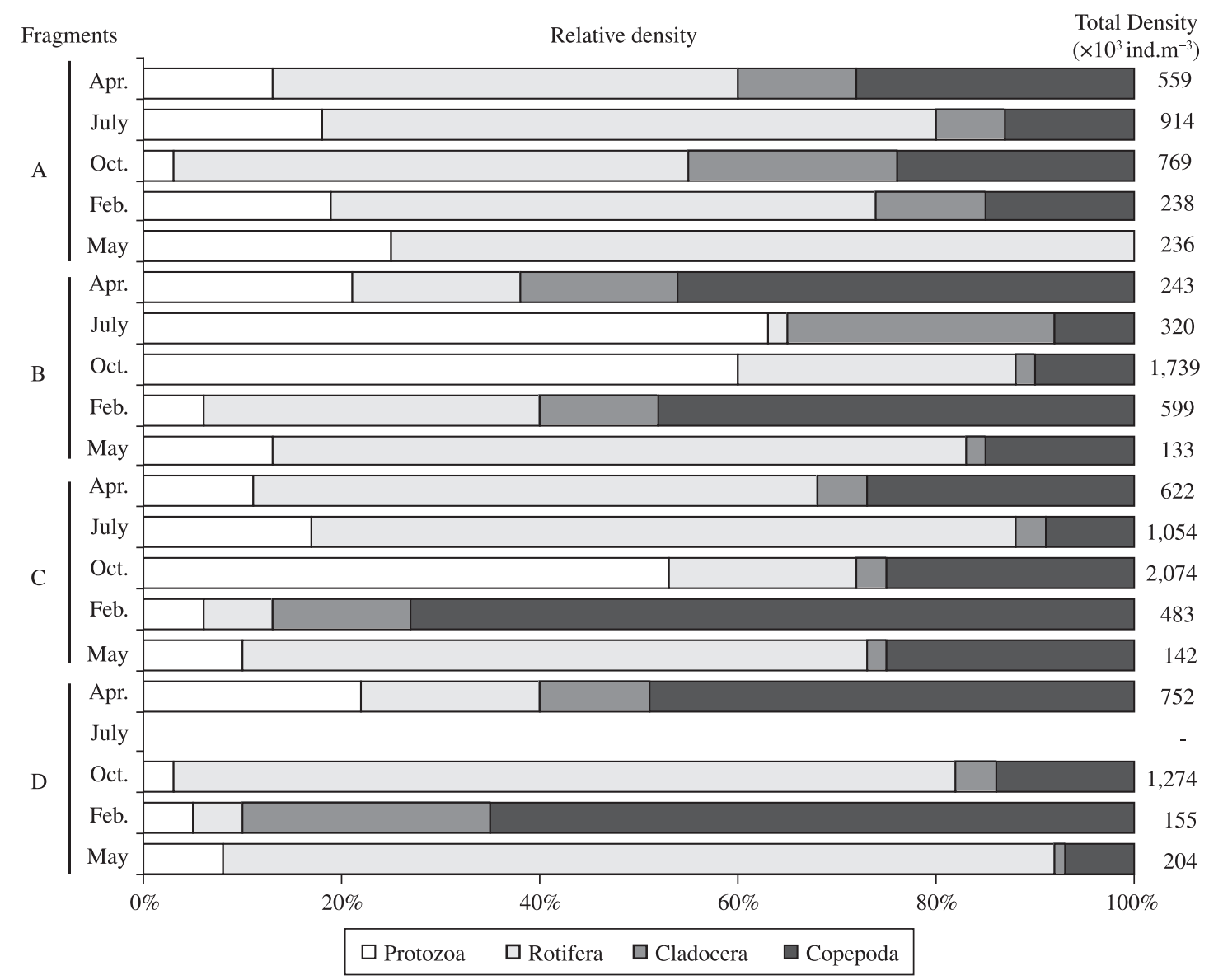

Figure 3. Relative (\%) and total density (the column at right side) of zooplankton at the four fragments (A to D) of the Coqueiral Lake at tri-monthly interval (April 2000 to May 2001).

Table 3. Taxa richness of zooplankton in the four fragments (A to D) and its sampling sites and temporal variability (from April 2000 to May 2001) in Coqueiral Lake.

\begin{tabular}{|c|c|c|c|c|c|c|}
\hline \multicolumn{2}{|c|}{ Fragments } & April & July & October & February & May \\
\hline \multicolumn{7}{|c|}{ A } \\
\hline \multirow[t]{4}{*}{ Site } & 1 & 13 & 21 & 17 & 28 & 26 \\
\hline & 2 & 17 & 20 & 16 & 36 & 12 \\
\hline & 3 & 23 & 17 & 17 & 35 & 16 \\
\hline & & $17.7 \pm 4.1$ & $19.3 \pm 1.7$ & $16.7 \pm 0.5$ & $33.0 \pm 3.6$ & $18.0 \pm 5.9$ \\
\hline \multicolumn{7}{|c|}{30.0 .0} \\
\hline \multirow[t]{5}{*}{ Site } & 4 & 22 & 0 & 0 & 40 & 15 \\
\hline & 5 & 16 & 8 & 20 & 34 & 12 \\
\hline & 6 & 16 & 1 & 19 & 37 & 19 \\
\hline & 7 & 17 & 0 & 0 & 33 & 16 \\
\hline & & $17.8 \pm 2.5$ & $2.3 \pm 3.3$ & $9.8 \pm 9.8$ & $36.0 \pm 2.7$ & $15.5 \pm 2.5$ \\
\hline \multicolumn{7}{|l|}{ C } \\
\hline \multirow[t]{4}{*}{ Site } & 8 & 19 & 16 & 11 & 22 & 10 \\
\hline & 9 & 16 & 17 & 14 & 26 & 15 \\
\hline & 10 & 16 & 0 & 27 & 35 & 14 \\
\hline & & $17.0 \pm 1.4$ & $11.0 \pm 7.8$ & $17.3 \pm 6.9$ & $27.7 \pm 5.4$ & $13.0 \pm 2.2$ \\
\hline \multicolumn{7}{|l|}{ D } \\
\hline \multirow[t]{2}{*}{ Site } & 11 & 14 & 1 & 22 & 33 & 15 \\
\hline & 12 & 16 & 0 & 26 & 19 & 12 \\
\hline \multicolumn{2}{|c|}{$\bar{x} \pm S D$} & $15.0 \pm 1.0$ & $0.5 \pm 0.5$ & $24.0 \pm 2.0$ & $26.0 \pm 7.0$ & $13.5 \pm 1.5$ \\
\hline
\end{tabular}


Table 4. Pearson correlations between attributes of zooplankton community and environmental variables for the four fragments of Coqueiral Lake $(* \mathrm{P}<0.05)$

\begin{tabular}{|c|c|c|c|c|c|}
\hline \multicolumn{2}{|c|}{ Relationships between } & \multicolumn{4}{|c|}{ Fragments } \\
\hline Attributes & Environmental variables & $\mathbf{A}$ & B & $\mathbf{C}$ & D \\
\hline \multirow[t]{7}{*}{ Taxon richness } & Mean temperature & $0.91 *$ & $0.93 *$ & $0.65 *$ & $0.93 *$ \\
\hline & Dissolved oxygen & $-0.72 *$ & $-0.52 *$ & -0.23 & -0.19 \\
\hline & Water transparency & $0.99 *$ & $0.78 *$ & $0.84 *$ & $0.60 *$ \\
\hline & Depth & $0.65^{*}$ & $0.66^{*}$ & 0.02 & 0.23 \\
\hline & $\mathrm{pH}$ & -0.18 & -0.25 & -0.35 & $0.89 *$ \\
\hline & Conductivity & 0.48 & $-0.79 *$ & 0.39 & $0.83^{*}$ \\
\hline & Chlorophyll- $a$ & 0.08 & 0.40 & -0.43 & -0.04 \\
\hline \multirow[t]{7}{*}{ Total abundance } & Mean temperature & $-0.93^{*}$ & $0.71 *$ & 0.05 & $0.50 *$ \\
\hline & Dissolved oxygen & $0.83^{*}$ & -0.36 & $0.70 *$ & 0.40 \\
\hline & Water transparency & $-0.80 *$ & -0.17 & -0.22 & -0.26 \\
\hline & Depth & $-0.92 *$ & -0.23 & $-0.87 *$ & -0.43 \\
\hline & $\mathrm{pH}$ & 0.28 & -0.12 & $0.94 *$ & 0.49 \\
\hline & Conductivity & $-0.57 *$ & 0.25 & $0.72 *$ & 0.38 \\
\hline & Chlorophyll- $a$ & $-0.56^{*}$ & 0.36 & $-0.77 *$ & -0.16 \\
\hline
\end{tabular}
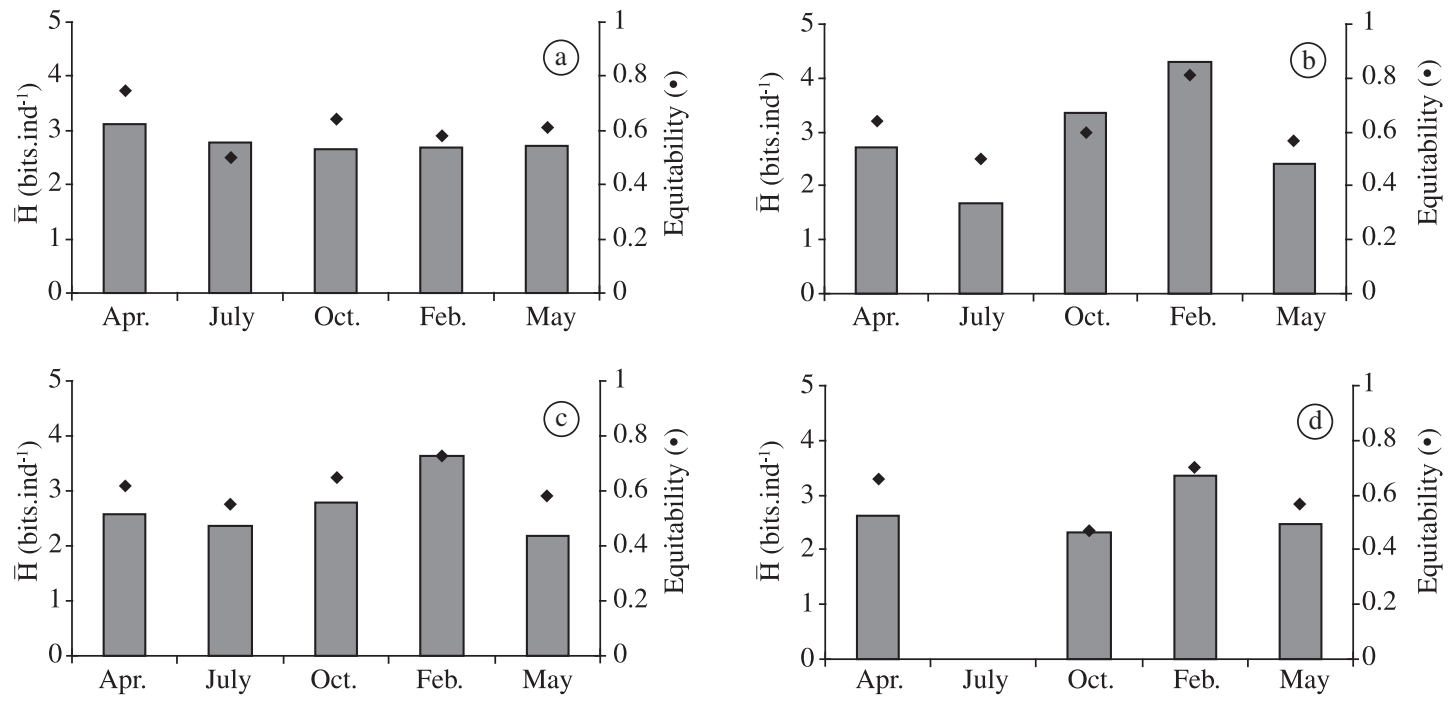

Figure 4. Temporal variability on Shannon Wiener diversity indexes $\left(\bar{H}\right.$, bits.ind $\left.{ }^{-1}\right)$ and equitability $(\bullet)$ of the zooplankton Community in the four fragments (a to d) of Coqueiral Lake.

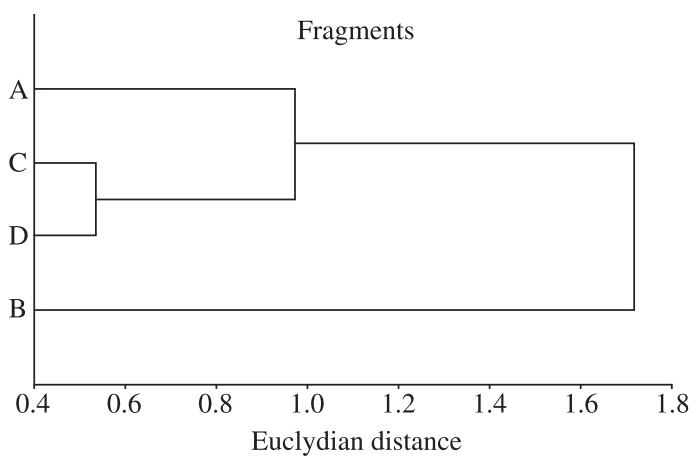

Figure 5. Similarity dendrogram of species richness of zooplankton between the four fragments (A to D) of Coqueiral Lake. in fragments A and C (Table 4). Positive correlation between water temperature and abundance was only found in fragments B and D (Table 4).

\section{Discussion}

The word "fragmentation" is used here to express the formation of water bodies isolated from one another by a reduction in area and volume of a large environment (Coqueiral Lake) due to the loss of hydrological connectivity of the lentic system with the river. In a situation prior to the drought, the original ecosystem (Coqueiral Lake) was a single water body, lateral to the river. In floodplains, the degree of fragmentation and connection between aquatic environments is affected by flood pulses 
which are very important in maintaining ecosystem integrity (Junk et al., 1989).

In the transition region between Paranapanema River and Jurumirim Reservoir, the adjacent lentic environments have different degrees of association with the water course (Henry, 2003). This zone - typical of a wetland presents no characteristics peculiar to a floodplain due to a permanent hydrologic connectivity between river and lakes, but during the year it is submitted to changes in water level and to operational management of the rainfall in reservoir (Henry, 2005). The prolonged drought during the study period (less than $50 \%$ of the rainfall in previous and following year, Henry, 2005) led to a loss of connection between the Coqueiral Lake and Paranapanema River. Evaporation and surface and hyporheic zone flux in the lake-to-river direction led to the formation of small isolated bodies of water (called fragments) in function of the bottom topography of Coqueiral Lake.

On a spatial scale, there were four different fragments compared to before fragmentation and after hydrological connection was recovered by the Paranapanema River. Fragment A was the deepest with lowest water transparency, dissolved oxygen, $\mathrm{pH}$, total alkalinity and conductivity. As annual variability of these factors in fragment A was lower than in the other fragments (De Nadai, 2006), it can be assumed that this fragment presents an apparent hydrological stability during the prolonged drought episode. During the year, fragment B had higher mean dissolved oxygen, total alkalinity, conductivity, and chlorophyll- $a$ concentration than the other fragments. Its specific position (near the connection point to the Paranapanema River, Figure 2) characterises the river's influence on its water quality, especially oxygen after river overflow. However, the shallow depth of this fragment before the inundation period produced a concentration effect which was confirmed by increasing conductivity values ( $>250 \mu \mathrm{S} . \mathrm{cm}^{-1}$ in July, August, and October, 2000; De Nadai, 2006; Henry et al., 2006). Different water transparency, $\mathrm{pH}$, and chlorophyll- $a$ conditions were found in fragments $\mathrm{C}$ and $\mathrm{D}$, the positions of which are different to the other two fragments by being further away from the Paranapanema River connection site, and by being shallower than fragment $\mathrm{C}$.

Two periods can be identified in temporal analysis of environmental factors: before the inundation period (April, July and October 2000), and the recovery period for hydrologic connectivity between the Paranapanema River and Coqueiral Lake (February and May 2001). In the first, dissolved oxygen decreased and water alkalinity and conductivity, and chlorophyll- $a$ increased over time demonstrating a concentration effect on the fragments proven by the reduction in their mean depths. In the second, a significant increase in fragment mean depths resulted in a drastic decrease in water oxygen, a reduction in $\mathrm{pH}$, total alkalinity, and chlorophyll- $a$. Temporal changes in these variables are due to recovery of the river-lake hydrological connectivity. The significant increase in depths at the different sites (fragments) caused plant submersion and growth in exposed sediment during the drought period. Conductivity was expected to increase and $\mathrm{pH}$ decrease due to degradation of submersed vegetation, but the lateral influx of water from the Paranapanema River into the Coqueiral Lake caused a dilution effect. According to Thomaz et al. (2007), the inundation pulse in floodplains has a homogenising effect on the water quality of marginal lakes with different hydrologic connectivity which is related to the distance from the main river channel. Despite the Paranapanema River lateral lakes being permanently connected to the water course (Henry, 2005), this prolonged drought episode led to different shaped fragments appearing by segmentation of the Coqueiral Lake after the hydrological pulse which is analogous to behaviour found in floodplains.

Size (area and volume) reduction of the four fragments with drought progression significantly affected zooplankton species richness, especially in July 2000. In this ninth month after disconnection of the Coqueiral Lake and Paranapanema River, a drastic reduction in depths caused sediment exposure at some sampling sites. Mean zooplankton species richness significantly decreased as a consequence of this reduction in colonization area (for instance fragments $\mathrm{C}$ and $\mathrm{D}$, see Table 3). Henry et al. (2006) also found reduced on phytoplankton richness in Coqueiral Lake (in a sampling site corresponding to fragment $\mathrm{B}$ ), dropping from 32 taxa in the second month after disconnection to 11 taxa after 11 months of lentic isolation. The low variability in zooplankton species richness in fragment $\mathrm{A}(\mathrm{CV}=7 \%)$ before the inundation period (April, July and October 2000) can be related to its apparent environmental stability, as there was only a slight mean depth reduction (three sites; from 1.1 to $0.7 \mathrm{~m}$, according to De Nadai, 2006) compared to the other fragments. The inundation pulse from December 2000 to February 2001, and the resulting recovery of the Paranapanema River - Coqueiral Lake hydrologic connectivity produced a $100 \%$ increase in the number of zooplankton species from sampling sites corresponding to the 4 fragments. The lateral influx of water from Paranapanema River into the lacustrine environment became a true ecological disturbance (measured by richness, diversity, and equitability) of zooplankton communities in the four isolated fragments. Except in fragment A sites, the increased zooplankton diversity and equitability in the other three fragments was attributed to hydrological pulse.

Several factors may have contributed to increased zooplankton species richness after lateral water influx from the Paranapanema River; one probable factor is species introduction by water course drift. According to Henry (2003), surveys prior to the prolonged drought showed maximum zooplankton species richness being about 2.7 times higher in the Paranapanema River than Coqueiral Lake. Ward et al. (1999) obtained higher planktonic crustacean richness in isolated lentic systems than in connected floodplain lacustrine ecosystems. 
In the Alto Paraná floodplain, Aoyagui and Bonecker (2004) recorded higher Rotifera richness in connected than in isolated lakes during the high water period; this suggests that the higher numbers of Rotifera species are related to the Paraná River connection. On the other hand, Galindo et al. (1994) found no relationship between micro-crustacean richness and inundated areas in Doñana National Park dune ponds (SW Spain). A second factor influencing increased zooplankton species in the pelagic zone of the four fragment sites will be washout from aquatic macrophytes after Paranapanema River inundation pulse. In fact, during the Coqueiral Lake drought and fragmentation period, an increase in area covered by Eichhornia azurea was seen (Henry, 2003). According to Afonso (2002), the relative micro-crustacean density range during the year varied from 7 to $21 \%$ of total living invertebrate density linked to E. azurea, with the highest relative density recorded during the high water period. Thus, the increase in zooplankton organisms in the Coqueiral Lake pelagic zone during the hydrologic pulse could probably be due to dispersion of the living fauna linked to aquatic macrophytes. A third factor could have been from emergence from diapausing eggs in sediment immediately after the lateral inundation. Invertebrate emergency from eggs or propagule banks in sediment appears to be an important tool for maintaining wetland ecological integrity (Angeler and Garcia, 2003). In a German floodplain, the hatching of resting Cyclopoida (Copepoda) eggs is an important survival strategy in temporarily connected environments (Frisch, 2002). Albritton and White (2004) have shown that higher numbers of Rotifera species and other zooplankton linked to the margin zone of a reservoir in the USA, hatched from sediment in areas regularly submitted to drought and inundation rather than from sites permanently covered by water. Although no information exists for the Coqueiral Lake, an experiment on hatching of resting eggs from sediment in another lacustrine environment isolated from the Paranapanema River, revealed a high number of organisms in diapause - nine Rotifera, four Cladocera, and one Copepoda hatched species (Panarelli et al., submitted). Thus, some of the species found after hydrological connection recovery probably hatched from egg banks on exposed Coqueiral Lake sediment.

An apparent concentration effect on total zooplankton density was seen in three of the four Coqueiral Lake fragments appearing during disconnection from the Paranapanema River. The significant increase in zooplankton abundance can be attributed to a reduction in colonization area and volume in the fragments with drought progression. The low variability $(\mathrm{CV}=24 \%)$ of total zooplankton organism density in fragment A can be related to its apparent hydrologic stability, similar to what occurred with species richness. A consequence of lateral water influx from the river was the reduction in total zooplankton density in the four fragments by a dilution effect. After the river lake connection was re-established, an increase in Copepoda relative density occurred in February 2000 in all sampling sites but those corresponding to fragment $\mathrm{A}$. The increase in relative copepod participation in the community after hydrological connectivity was re-established could be due to washout of micro-crustaceans living on E. azurea stands, species hatching from eggs in sediment areas corresponding to the three fragments, and to organisms drifting in on water flux from the Paranapanema River, even though relative Copepoda abundance in the water course corresponds to $<10 \%$ total zooplankton organism abundance in the increasing water period (Panarelli et al., 2003).

The more distant fragments $(\mathrm{C}$ and $\mathrm{D})$ were similar for zooplankton species richness and total density; fragment A showed an intermediate response, and fragment B had higher dissimilarity than the others (Figure 5). This fragment is directly influenced by river action, as was also seen during hydrological connection recovery (Casanova, 2004). In fragment A, with an apparent hydrological stability even during the drought period, total zooplankton abundance increase is influenced by reduced abiotic factor values (except $\mathrm{pH}$ ), while increased water temperature and depth apparently caused an increase in species richness. Water transparency appears to be a favourable controlling factor for increased species richness, despite the reduction in fragment size during drought progression leading to sediment re-suspension during windy episodes.

Before Coqueiral Lake disconnection from the Paranapanema River, mean total density was lower than in this study (Martins and Henry, 2004; $143 \times 10^{3} \pm$ $20.9 \times 10^{3}$ individuals. $\mathrm{m}^{-3} ; \mathrm{CV}=15 \%$, mean of three sampling periods from a site corresponding to fragment B). However, Rotifera organism relative participation ( $87 \%$ of total community) was completely distinct from this study (Figure 2).

In relation to specific zooplankton composition, nine of the taxa recorded before the inundation period (April, July and October, 2000), including one Protozoa (Centropyxis sp.), three Rotifera (Hexarthra sp., Keratella cochlearis and Plationus patulus), one Copepoda (Mesocyclops sp.), and four Cladocera (Cerodaphnia cornuta, Diaphanosoma brevireme, D. birgei and Macrothrix spinosa) were not found in May, 2001 (the after inundation period). On the other hand, eleven taxa not recorded before the inundation period appeared after the inundation period, two Protozoa (Arcella discoides and Difflugia gramen) and the rest from Rotifera (Bdelloidea, Brachionus angularis, Cephallodela sp., Epiphanes sp., Filinia terminalis, Lecane sp., L. cf. furcata, L. hamata and Trichocerca similis). Despite the apparent resilience of total zooplankton taxa richness (comparing before and after inundation, see Table 3 ), a change in specific composition was evident. The alteration in zooplankton community component composition was due to a disturbance factor (the inundation pulse from December 2000 to February 2001). Introduction by water drift from the Paranapanema River and removal of taxa commonly linked to aquatic macrophytes (for instance, species of 
Lecane sp.) can be modifying factors for of zooplankton community composition.

In conclusion, a concentration effect on zooplankton abundance was recorded with drought progression, but without significant modifications in species richness and diversity. After the Paranapanema River inundation pulse, a reduction in total zooplankton density was found due to the dilution effect, but a significant increase in taxa richness and diversity was also observed. The lateral influx of water acted as a temporary disturbance factor on community structure, but specific composition also presented some alterations.

Acknowledgements - This research received financial support from FAPESP (Proc. 97/04999-8) and CAPES (scholarship grant to R. De Nadai). The authors are also grateful to A. C. Pião, H. A. Rodrigues, C. E. Knaggs, and J. Ramos for help with statistical analysis, field sampling, English language revision and manuscript preparation, respectively.

\section{References}

AFONSO, AAO., 2002. Relações da fauna associada à Eichhornia azurea (Swartz) Kunth com as variáveis abióticas em lagoas laterais de diferentes graus de conexão ao Rio Paranapanema (zona de desembocadura na Represa de Jurumirim, SP). Botucatu: Instituto de Biociências, UNESP. [Tese de Doutorado].

ALBRITTON, CJ. and WHITE, DS., 2004. Hatching of rotifer eggs from reservoir sediment. Southeastern Naturalist, vol. 3, no. 2 , p. 359-370

ANGELER, DG. and GARCIA, G., 2003. Using emergence from soil propagule banks as indicators of ecological integrity in wetlands: advantages and limitations. Journal of North American Benthological Society, vol. 24, no. 4, p. 740-752.

AOYAGUI, ASM. and BONECKER, CC., 2004. Rotifers in different environments of the Upper Paraná floodplain (Brazil): richness, abundance and the relationship with connectivity. Hydrobiologia, vol. 522, no. 1/3, p. 281-290.

BOIX, D., SALA, J. and MORENO-AMICH, R., 2001. The faunal composition of Espolla Pond (NE Iberian Peninsula): the neglected biodiversity of temporary waters. Wetlands, vol. 21, no. 4 , p. $577-592$.

CASANOVA, SMC., 2005. Análise da estrutura da comunidade zooplanctônica na região de desembocadura do Rio Paranapanema na Represa de Jurumirim (SP), com ênfase na dinâmica populacional de Rotifera. Botucatu: Instituto de Biociências, UNESP. [Tese de Doutorado].

DAJOZ, R., 1973. Ecologia Geral. Petrópolis: Vozes. 472 p.

EDMONDSON, WT., 1959. Freshwater biology. 2 ed. London: John Wiley \& Sons. 1248 p.

FRISCH, D., 2002. Dormancy, dispersal and the survival of cyclopoids copepods (Cyclopoida, Copepoda) in a lowland floodplain. Freshawater Biology, vol. 47, no. 7, p. 1269-1281.

GALAT, DL., KUBIASIAK, JF., HOOKER, JB. and SOWA, LM., 1997. Geomorphology, distribution and connectivity of lower Missouri River floodplain waterbodies scoured by the flood of 1993. Internationale Vereinigung für theoretische und angewandte Limnologie, vol. 26, Part 2, p. 869-878.
GALINDO, MD., MATA, AJ., MAZUELOS, N., and SERRANO, L., 1994. Microcrustaceans and rotifer diversity and richness relating to water temoporality in dune ponds of the Doñana National Park (SW Spain). Internationale Vereinigung für theoretische und angewandte Limnologie, vol. 25, Part 3, p. 1350-1356.

GOLTERMAN, HL. and CLYMO, RS., 1969. Methods for chemical analysis of Freshwaters. Oxford: Blackwell Scientific Publications. 166 p. (IBP Handbook no. 8).

HENRY, R., 2005. The connectivity of the Paranapanema River with two lateral lakes in its mouth zone into Jurumirim Reservoir. Acta Limnologica Brasiliensia, vol. 17, no. 1, p. 57-69.

HENRY, R., 2003. Os ecótonos nas interfaces dos ecossistemas aquáticos: conceitos, tipos, processos e importância. Estudo de aplicação em lagoas marginais ao Rio Paranapanema na zona de sua desembocadura na Represa de Jurumirim. In HENRY, R. (Org.). Ecótonos nas interfaces dos ecossistemas aquáticos. São Carlos: Rima Editora, p. 1-28.

HENRY, R., PANARELLI, EA., CASANOVA, SMC., SUIBERTO, MR. and AFONSO, AAO., 2006. Interações hidrológicas entre lagoas marginais e o Rio Paranapanema na zona de sua desembocadura na Represa de Jurumirim. In NOGUEIRA, MG., HENRY, R. and JORCIN, A. (Org.). Ecologia de reservatórios: impactos potenciais, ações de manejo e sistemas em cascatas. São Carlos: Rima Editora. p. 57-82.

HENRY, R., USHINOHAMA, E. and FERREIRA, RMR., 2006. O fitoplâncton em três lagoas e no Rio Paranapanema na zona de sua desembocadura na Represa de Jurumirim durante um período prolongado de seca. Rev. Bras. Bot. vol. 29, no. 3, p. $399-414$

KOROVCHINSKY, MM., 1992. Guides to identification of the microinvertebrates Sididae \& Holopeidae (Crustacea: Daphniiformes). The Hague. SPB Academic Publishing. 42 p.

KOSTE, W., 1978. Rotatoria: die radertiere mitteleuropas. [S.l.]: [s.n.]. 237 p. Vol. I e II. Text Band.

KREBS, CJ., 1972. Ecology: the experimental analysis of distribution and abundance. New York: Harper \& Row Publishers. $694 \mathrm{p}$.

LOUREIRO, LMAE., 1997. Manual de identificação de cladóceros límnicos do Brasil. Brasília: Editora Universia. $156 \mathrm{p}$.

MARTINS, GM. and HENRY, R., 2004. Composição e abundância do zooplâncton em três lagoas laterais ao Rio Paranapanema na zona de sua desembocadura na Represa e Jurumirim (São Paulo). In CHELLAPPA, NT., CHELLAPPA, B. and PASSAVANTE, JZO. (Ed.). Ecologia tropical aquática. Natal: ServGraf. p. 53-72.

MOULTON, TP. and SOUZA, ML., 2006. Conservação com base em bacias hidrográficas. In ROCHA, CFD., BERGALLO, HG., van SLUYS, M., and ALVES, MAS. (Ed.). Biologia da conservação: essências. São Carlos: Rima Editora. p. 157-181.

NADAI, R., 2006. Distribuição horizontal e vertical da comunidade zooplanctônica, nos períodos de seca e chuva, em ambientes lacustres na área de transição Rio Paranapanema Represa de Jurumirim, São Paulo, Brasil. Botucatu: Instituto de Biociências, UNESP. [Dissertação de Mestrado]. 240 p.

OLIFIERS, N. and CERQUEIRA, R., 2006. Fragmentação de habitat: efeitos históricos e ecológicos. In ROCHA, CFD., BERGALLO, HV., van SLUYS, M. and ALVES, MAS. (Ed.). 
Biologia da conservação: essências. São Carlos: Rima Editora. p. 261-279.

PANARELLI, E., CASANOVA, SMC., NOGUEIRA, MG., MITSUKA, PM. and HENRY, R., 2003. A comunidade zooplanctônica ao longo de gradientes longitudinais no Rio Paranapanema/Represa de Jurumirim (São Paulo, Brasil). In HENRY, R. (Org.). Ecótonos nas interfaces dos ecossistemas aquáticos. São Carlos: Rima Editora. p. 129-160.

ROCHA, CFD., BERGALLO, HG., van SLUYS, M., ALVES, MAS. and JENKINS, C., 2006. Corredores ecológicos e conservação da biodiversidade: um estudo de caso na Mata Atlântica. In: ROCHA, CFD., BERGALLO, HG., van SLUYS, M. and ALVES, MAS. (Ed.). Biologia da Conservação: essências. São Carlos: Rima Editora. p. 317-342.

ROCHA, O. and MATSUMURA-TUNDISI, T., 1976. Atlas do zooplâncton: represa do Broa. São Carlos: UFSCar. 68 p.

SAUNDERS, DA., HOBBS, RJ. and MARGULES, CR., 1991. Biological consequences of ecosystem fragmentation: a review. Conservation Biology, vol. 5, no. 1, p.18-32.
SENDACZ, S. and MONTEIRO JUNIOR, AJ., 2003. Zooplâncton e características limnológicas da planície de inundação do Rio Paraná. In HENRY, R. (Org.). Ecótonos nas interfaces dos ecossistemas aquáticos. São Carlos: Rima Editora. p. 61-82.

SHELDON, F., BOULTON, AJ. and PUCKRIDGE, JT., 2002. Conservation value of variable connectivity: aquatic invertebrate assemblages of channel and floodplain habitats of a Central Australian arid-zone river, Cooper Creek. Biological Conservation, vol. 103, no. 1, p. 13-31.

THOMAZ, SM., BINI, LM. and BOZELLI, RL., 2007. Floods increase similarity amoung aquatic habitats in river-floodplain systems. Hydrobiologia, vol. 579, no. 1, p. 1-13.

VALENTIN, JL., 2000. Ecologia numérica: uma introdução à análise multivariada de dados ecológicos. Rio de Janeiro: Editora Interciência. 117 p.

WARD, JV., TOCKNER, K. and SCHIEMER, F., 1999. Biodiversity of floodplain river ecosystems: ecotones and connectivity. River Research and Applications, vol. 15, no. 1-3, p. $125-139$. 